\title{
Triaxial AFM Probes for Noncontact Trapping and Manipulation
}

\section{Citation}

Brown, Keith A., and Robert M. Westervelt. 2011. Triaxial AFM probes for noncontact trapping and manipulation. Nano Letters 11(8): 3197-3201.

\section{Published Version}

doi:10.1021/nl201434t

\section{Permanent link}

http://nrs.harvard.edu/urn-3:HUL.InstRepos:9453698

\section{Terms of Use}

This article was downloaded from Harvard University's DASH repository, and is made available under the terms and conditions applicable to Open Access Policy Articles, as set forth at http:// nrs.harvard.edu/urn-3:HUL.InstRepos:dash.current.terms-of-use\#OAP

\section{Share Your Story}

The Harvard community has made this article openly available.

Please share how this access benefits you. Submit a story.

Accessibility 


\title{
Triaxial AFM probes for non-contact trapping and manipulation
}

\author{
Keith A. Brown and Robert M. Westervelt* \\ School of Engineering and Applied Sciences, and Department of Physics, \\ Harvard University, Cambridge, MA 02138 \\ *e-mail: westervelt@seas.harvard.edu
}

\begin{abstract}
:
We show that a triaxial AFM probe creates a non-contact trap for a single particle in a fluid via negative dielectrophoresis (nDEP). A zero in the electric field profile traps the particle above the probe surface, avoiding adhesion, and the repulsive region surrounding the zero pushes other particles away, preventing clustering. Triaxial probes are promising for three-dimensional assembly and for selective imaging of a particular property of a sample using interchangeable functionalized particles.
\end{abstract}

Keywords: Dielectrophoresis, Non-contact trapping, AFM, Assembly, Nanoparticles, Triaxial, 
The atomic force microscope (AFM) is an indispensable tool for manipulating and imaging nanoscale materials. ${ }^{1-3}$ AFM tips have been used to pattern and assemble small objects by pushing and dragging, ${ }^{4,5}$ scanned probe lithography, ${ }^{6,7}$ and by lifting with two coordinated AFM probes. ${ }^{8}$ In addition to applications in assembly, manipulating objects with an AFM tip allows new types of imaging by using an attached particle to provide a specific tip-sample interaction. Examples of functionalization-interaction pairs include silica beads to measure colloidal forces, ${ }^{9}$ biological cells for measuring cell-cell interactions,,${ }^{10}$ metallic nanoparticles for scanning near field optical microscopy ${ }^{11}$ and carbon nanotubes ${ }^{12}$ and nanoneedles ${ }^{13}$ for high resolution imaging. Single particle manipulation is limited by clustering of many particles and the "sticky finger" problem in which nanoscale particles become irreversibly stuck to surfaces or the AFM probe. ${ }^{14}$

An atomic force microscope (AFM) probe that can grab and release particles without the limitations of stiction and clustering would have many applications in manipulation and imaging. The ability to pick-and-place single nanoscale particles for three-dimensional assembly could enable the construction of interacting quantum systems. ${ }^{15}$ A single particle held by the probe would allow selective imaging with a functionalized material, as in optical tweezing, ${ }^{15,16}$ and has great promise for single-molecule force spectroscopy. ${ }^{3}$

We recently created coaxial AFM probes that can perform pick-and-place manipulation of microscale objects, and can image with a particle trapped at their tip. ${ }^{17}$ Coaxial probes use positive dielectrophoresis (pDEP), the attraction of an induced dipole towards regions of high electric field, which is widely used in nanotechnology, ${ }^{18}$ biotechnology, ${ }^{19}$ and single particle trapping. ${ }^{20}$ A coaxial probe consists of a conducting AFM tip surrounded by a grounded shell that produces a sharp maximum in the electric field $E$ at the tip's end. ${ }^{17,21,22}$ Drawbacks of this 
approach are clustering of multiple particles, and stiction of attached particles as they must be held in contact. ${ }^{14,23}$

In this paper, we present triaxial AFM probes and demonstrate non-contact trapping of nanoscale objects via negative dielectrophoresis (nDEP). ${ }^{24}$ The tip of a triaxial probe (Figure 1a) shows three electrodes: a conducting core surrounded by an inner and an outer conducting shell, highlighted in Figure 1b. By applying a voltage to the inner shell electrode, the triaxial probe creates an electric field profile $E$ with a zero at a height $z_{T}$ above the end of the probe, as shown by the electrostatic simulation in Figure 1c. Objects that are less polarizable than the fluid medium are trapped in the electric-field zero at $z_{T}$, which we designate as the nDEP trap. The trap is physically smaller than the triaxial tip, promising for the manipulation of nanoscale particles. We can hold a $100 \mathrm{~nm}$ radius polystyrene sphere in a region commensurate with its size using a triaxial probe with tip diameter $\sim 7 \mu \mathrm{m}$, much larger than the nanoparticle. By adjusting the voltage on the core electrode $\left(V_{l}\right)$ relative to the voltage on the inner shell electrode $\left(V_{2}\right)$, the trap can be opened and closed, and the height $z_{T}$ of the trapped particle above the tip can be adjusted, allowing the user to position and assemble particles.

Figure 2 illustrates how polystyrene beads are drawn toward and trapped by the triaxial probe. Triaxial electrodes are nanofabricated onto commercial AFM probes (see Supporting Information). A probe is held in a suspension of fluorescent polystyrene beads with radius $a=100 \mathrm{~nm}$, suspended in a 1:1 v/v mixture of deionized water : glycerol and observed with a fluorescence microscope (see Supporting Information). The way the polystyrene beads interact with a radio frequency (RF) electric field $E$ depends on the frequency $f$ of $E$ (see Supporting Information) ${ }^{25-27}$ In particular, these beads exhibit a crossover frequency $f_{C} \approx 800 \mathrm{kHz}$ such that if $f<f_{C}$, their surface conductance increases their polarizability sufficiently so that they 
experience $\mathrm{pDEP}$ and are pulled to regions of high electric field. Conversely, if $f>f_{C}$, they will be repelled from regions of high field with nDEP. An attractive field profile (Figure 2a) is created by applying $10 \mathrm{~V}$ peak-to-peak to the inner shell at a low frequency $(f=100 \mathrm{kHz})$ while grounding the core and outer shell electrodes. The sequence of images shown in Figures $2 b-f$, taken at $200 \mathrm{~ms}$ intervals, show how beads are attracted toward the high field region near the triaxial tip - the bead marked by an arrow moves towards the probe, and becomes trapped on its surface in Figure 2f.

By increasing the RF frequency to $f=5 \mathrm{MHz}$, we turn on the nDEP trap above the triaxial probe. The polystyrene beads are now repelled from high field regions - some are drawn into the $\mathrm{nDEP}$ trap and the rest are pushed out into the medium, as shown in Figure $2 \mathrm{~g}$. Before $f$ is increased (Figure 2h), many beads are attached to the probe surface. Just after the increase (Figure 2i), the beads on the probe are jettisoned. In the subsequent frames (Figures $2 \mathrm{j}-1$ ), the majority of the beads are pushed away from the probe, while those marked with the red arrow are pushed to the electric field zero at the center of the nDEP trap.

Figure 3 shows the triaxial probe holding a polystyrene bead in the nDEP trap within a region comparable to the size of the bead. Figure $3 \mathrm{a}$ is a sequence of images that show the motion of a bead (red) trapped above the triaxial probe, and a bead (blue) adhered to the probe surface. The distribution of trapped and adhered bead positions (Figure 3b) is found by fitting two-dimensional Gaussians to the beads in each image. The position of the trapped bead relative to the probe (Figure 3c), is found by comparing the positions of the trapped and adhered bead in each image. Principle component analysis is used to find the tight $\left(x^{\prime}\right)$ and loose $\left(y^{\prime}\right)$ axes of the spatial distribution over time. The strength of the nDEP trap is represented by histograms of the displacements along the $x^{\prime}$ and $y^{\prime}$ axes, shown in Figures $3 \mathrm{~d}$ and $3 \mathrm{e}$. The spatial distributions are 
fit very well by Gaussians, appropriate for thermal fluctuations against a linear restoring force. ${ }^{28}$ The Gaussian fits provide standard deviations $\sigma_{x^{\prime}}=133 \mathrm{~nm}$ and $\sigma_{y^{\prime}}=204 \mathrm{~nm}$ that are comparable to the $100 \mathrm{~nm}$ radius of the bead.

The measured shape and strength of the nDEP trap are in agreement with those predicted by theoretical simulations. The magnitude of the thermal fluctuations in the bead's position can be estimated from the triaxial probe geometry, the dielectric properties of the system, and our theoretical model for triaxial probes. ${ }^{24}$ For an ideal triaxial tip, this analysis (see Supporting Information) predicts standard deviations $\sigma_{z}=66 \mathrm{~nm}$ and $\sigma_{r}=102 \mathrm{~nm}$ in the axial and radial directions respectively, comparable to our observations. The experimental standard deviations and the observed DEP velocities (see Supporting Information) are both consistent with the values of $V_{1}$ and $V_{2}$ being attenuated to $\sim 50 \%$ of the applied values. The ratio $\sigma_{x^{\prime}} / \sigma_{y^{\prime}}=0.65$ of the experimental standard deviations, which is a measure of the trap shape, is in excellent agreement with the theoretically determined ratio $\sigma_{z} / \sigma_{r}=0.64$, indicating that the shape of the trap matches our theoretical predictions.

Figure 4 demonstrates that we can precisely control the height $z_{T}$ of the trap above the probe surface by changing the voltages on the core and shell electrodes. By setting $V_{l}=V_{B}$ $\sin (2 \pi f t)$ and $V_{2}=\left[V_{B}+\Delta V\right] \sin (2 \pi f t)$, we make $z_{T}$ a monotonic function of the control voltage $V_{B}$ (see Supporting Information). Figures $4 \mathrm{a}$ and $4 \mathrm{~b}$ show images of a single trapped fluorescent polystyrene bead for $V_{B}=0 \mathrm{~V}$ and $V_{B}=-\Delta V / 2$ respectively, with bead radius $a=460 \mathrm{~nm}$, $\Delta V=7.5 \mathrm{~V}$, and $f=5 \mathrm{MHz}$. The positions of a bead trapped in the $\mathrm{nDEP}$ trap (red $\mathrm{X})$ and a bead adhered to the back of the probe (blue X) are found with two-dimensional Gaussian fitting. The movement of the trapped bead relative to the probe is found for each pair of beads. As $V_{B}$ is 
swept from $0 \mathrm{~V}$ in Figure $4 \mathrm{a}$ to $-\Delta V / 2$ in Figure $4 \mathrm{~b}$, the bead moves away from the tip. By driving $V_{B}$ sinusoidally at $2 \mathrm{~Hz}$, the periodic motion of the bead may be tracked (Figure 4c). The height $z_{T}$ of the trap center relative to the tip (Figure 4d), is a smooth and monotonic function of $V_{B}$, allowing the user to precisely control the height of a trapped bead above the triaxial probe surface. By changing $V_{B}$ from $0 \mathrm{~V}$ to $-\Delta V / 2$, the bead is moved $\sim 1 \mu \mathrm{m}$. A straight line (red) fits the trap motion well with a slope $-270 \mathrm{~nm} / \mathrm{V}$, in agreement with the theoretical estimate $\sim-200 \mathrm{~nm} / \mathrm{V}$ (see Supporting Information). The residual (Figure 4e), fit by a Gaussian, gives a standard deviation in trap height of only $54 \mathrm{~nm}$ about the average value, much smaller than the $460 \mathrm{~nm}$ radius of the bead.

Triaxial probes are well suited to pick-and-place nanoassembly, because the dimensions of the trap are much smaller than the tip ${ }^{24}$, as shown in Figure 1c. A relatively large tip and bead size were chosen for this experiment to allow optical imaging, but much smaller dimensions are possible. The trap's electric field pattern, shown in Figure 1c, is universal; if the scale bar and the tip voltage are scaled down by the same factor, the pattern of the electric field and its amplitude will remain precisely the same. The limit on how tightly an object can be held is determined by dielectric breakdown of the medium. Simulations show that a Si sphere with radius $a=5 \mathrm{~nm}$ can be trapped at room temperature by a triaxial tip with radius $300 \mathrm{~nm}$ (see Supporting Information). Smaller objects can be trapped by increasing the tip voltage or by reducing the tip radius $R$. The standard deviation $\sigma$ in particle position at room temperature scales as $\sigma$ ? $a^{3 / 2} V_{r m s}{ }^{-1} R^{2}$ where $a$ is the particle radius, $V_{r m s}$ is the root mean square voltage, and $R$ is the electrode spacing. In this experiment, we measure $\sigma \leq 200 \mathrm{~nm}$ for $a=100 \mathrm{~nm}$, $R=1.6 \mu \mathrm{m}$, and $V_{r m s}=5.3 \mathrm{~V}$. By increasing $V_{r m s}$ by a factor of 25 , the scaling relation predicts that we can achieve a smaller standard deviation $\sigma \leq 8 \mathrm{~nm}$ without risking dielectric breakdown. 
A sharper probe with $R=64 \mathrm{~nm}$ can trap a smaller nanoparticle with $\mathrm{a}=10 \mathrm{~nm}$ in a space smaller than its radius, $\sigma<a$, for the same tip voltage $V_{r m s}=5.3 \mathrm{~V}$.

In this paper, we have shown that a triaxial AFM probe provides a non-contact approach for trapping a single nanoparticle at room temperature. Triaxial probes are strong candidates for pick-and-place assembly of nanoscale objects. Particles much smaller than the end of the triaxial probe can be grabbed out of a suspension using negative dielectrophoresis (Figure 2) and held in a potential well comparable to their size (Figure 3 and Video S1). The trapped particle can then be moved to the desired location (Video S3). The trapped particle can then be placed by pushing it away from the probe end by adjusting the tip voltages (Figure 4 and Video S2), or released into suspension by turning off the trapping field (Video S4). This pick-and-place technique works for particles as small as $5 \mathrm{~nm}$ at room temperature, and is promising for the construction of quantum devices. ${ }^{5}$ The triaxial probe presented here is compatible with commercial AFMs, and the full capabilities of a triaxial probe will be realized by leveraging nanometer scale positioning and force-measurement afforded by the AFM apparatus. Dielectrophoresis has been used for imaging using conventional AFM probes ${ }^{29}$ and with coaxial AFM probes ${ }^{30}$ for enhanced spatial resolution. Triaxial probes offer more control of the electric field distribution and new opportunities for imaging. For a system with a nonlinear response, the electric field zero above a triaxial probe can provide high spatial resolution images in analogy with nonlinear optical stimulated emission depletion (STED) microscopy. ${ }^{31}$

\section{Acknowledgements}


We acknowledge Evangelos Gatzogiannis for assistance with the optical microscope and Jim MacArthur help with electronics. We acknowledge support by the Department of Defense through a National Defense Science \& Engineering Graduate (NDSEG) Fellowship, the National Cancer Institute MIT-Harvard Center of Cancer Nanotechnology Excellence, and the Department of Energy under grant DE-FG02-07ER46422.

\section{Supporting Information Available}

Additional information is available on the theory of DEP, measuring velocity of nanoparticles near a triaxial probe (Figure S1), the electrostatic model of a triaxial probe (Figure S2 and S3), details of triaxial probe fabrication, and a description of the apparatus (Figure S4). Four videos are available that demonstrate the trapping of nanoparticles with triaxial probes, Video S1 depicts the Brownian motion of a $100 \mathrm{~nm}$ radius bead in the $\mathrm{nDEP}$ trap and Video $\mathrm{S} 2$ depicts moving a $460 \mathrm{~nm}$ radius bead relative to the tip. Video $\mathrm{S} 3$ shows holding a $100 \mathrm{~nm}$ radius bead while moving the triaxial probe with the three axis controller. Video S4 depicts beads being released back into suspension by turning off the trapping field. This material is available free of charge via the Internet at http://pubs.acs.org

\section{References}

1. Giessibl, F. J. Rev. Mod. Phys. 2003, 75, 949-983.

2. Benstetter, G.; Biberger, R.; Liu, D. Thin Solid Films 2009, 517, 5100-5105.

3. Müller, D. J.; DuFrêne, Y. F. Nature Nanotechnology 2008, 3, 261-269.

4. Requicha, A. A. G. Proc. IEEE 2003, 91, 1922-1933.

5. Barth, M.; Nüsse, N.; Löchel, B.; Benson, O. Optics Letters 2009, 34, 1108-1110.

6. Tseng, A. A.; Notargiacomo, A.; Chen, T. P. J. Vac. Sci. Technol. B 2005, 23, 877-894.

7. Salaita, K.; Wang, Y.; Mirkin, C. A. Nat. Nanotechnol. 2007, 2, 145-155. 
8. Xie, H.; Haliyo, D. S.; Régnier, S. A. Nanotechnology 2009, 20, 215301.

9. Ducker, W. A.; Senden, T. J.; Pashley, R. M. Nature 1991, 353, 239-241.

10. Lower, S. K.; Tadanier, C. J.; Hochella, M. F. Jr. Geochim. Cosmochim. Acta 2000, 64, 3133-3139.

11. Gan, Y. Rev. Sci. Instrum. 2007, 78, 081101.

12. Hafner, J. H.; Cheung, C.-L.; Woolley, A. T.; Lieber, C. M. Prog. Biophys. Mol. Biol. 2001, 77, 73-110.

13. Yazdanpanah, M. M.; Harfenist, S. A.; Safir, A.; Cohn, R. W. J. Appl. Phys. 2005, 98, 073510.

14. Komvopoulos, K. J. Adhesion Sci. Technol. 2003, 17, 477-517.

15. Moffitt, J. R.; Chemla, Y. R.; Smith, S. B.; Bustamante, C. Annu. Rev. Biochem. 2008, 77, 205-228.

16. Akerlof, G. J. Am. Chem. Soc. 1932, 54, 4125-4139.

17. Brown, K. A.; Aguilar, J. A.; Westervelt, R. M. Appl. Phys. Lett. 2010, 96, 123109.

18. Hughes, M. P. Nanotechnology 2000, 11, 124-132.

19. Voldman, J. Annu. Rev. Biomed. Eng. 2006, 8, 425-454.

20. Zheng, L.; Brody, J. P.; Burke, P. J. Biosens. Bioelectron. 2004, 20, 606-619.

21. Rosner, B. T.; Bork, T.; Agrawal, V.; Weide, D. W. v. d. Sens. Actuators, A 2002, 102, 185-194.

22. Noh, J. H.; Nikiforov, M.; Kalinin, S. V.; Vertegel, A. A.; Rack, P. D. Nanotechnology 2010, 21, 365302 .

23. Jones, T. B.; Bliss, G. W. J. Appl. Phys. 1977, 48, 1412-1417.

24. Brown, K. A.; Westervelt, R. M. Nanotechnology 2009, 20, 385302.

25. Akerlof, G. J. Am. Chem. Soc. 1932, 54, 4125-4139.

26. Weast, R. C. Handbook of Chemistry and Physics. 67th Ed.; CRC Press Inc.: Boca Raton, 1986.

27. Cui, L.; Holmes, D.; Morgan, H. Electrophoresis 2001, 22, 3893-3901.

28. Reif, F. Fundamentals of statistical and thermal physics; McGraw-Hill Inc.: Boston, 1965.

29. Hilton, A. M.; Lynch, B. P.; Simpson, G. J. Anal. Chem. 2005, 77, 8008-8012.

30. Brown, K. A.; Berezovsky, J.; Westervelt, R. M. Appl. Phys. Lett. 2011, 98, 183103. 
31. Willig, K. I.; Harke, B.; Medda, R.; Hell, S. W. Nat. Meth. 2007, 4, 915-918.

\section{Figures}
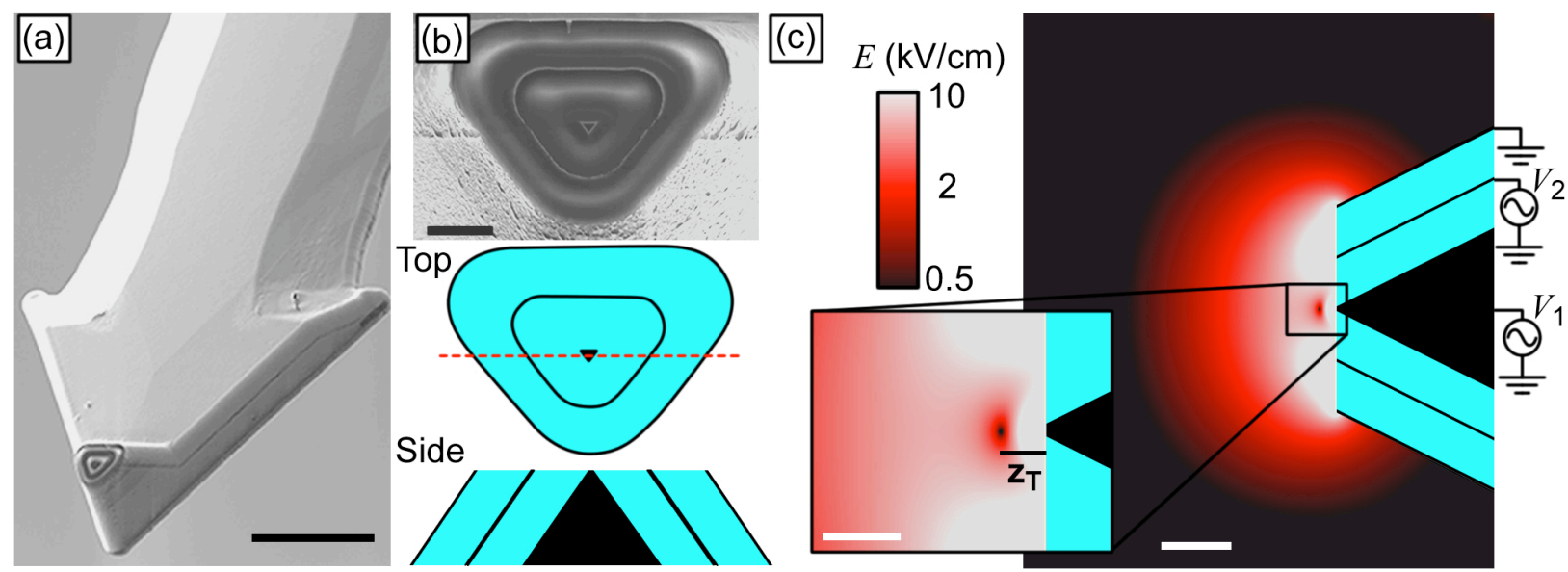

FIGURE 1. A triaxial AFM probe and its electric field showing the nDEP trap. (a) Scanning electron micrograph of a triaxial AFM probe. The triaxial electrodes are visible at the bottom of the image and the cantilever extends off the top of the image. The scale bar is $20 \mu \mathrm{m}$.

(b) Magnified view of the triaxial electrodes. The light triangle at the center is the original AFM probe that makes up the core conductor. The inner shell electrode is visible as a thin line around the core. The outer shell is visible at the edge of the electrodes. The scale bar is $2 \mu \mathrm{m}$. In the schematic diagram of the triaxial probe tip below, the electrodes are black and the insulating layers are blue. The dashed line shows the location of the side-view cross section shown in Figure 1c. (c) Axisymmetric simulation (Maxwell 2D - Ansys) of the electric field magnitude $E$ field near the tip of the triaxial probe with $V_{l}=0$ and $V_{2}=10 \mathrm{~V}$. The field decays quickly away from the tip and there is an electric field zero visible displaced from the surface of the probe. The scale bar is $2 \mu \mathrm{m}$. The inset shows a magnification of the trapping region. The electric field zero is visible a distance $z_{T}$ from the surface of the probe. The scale bar of the inset is $400 \mathrm{~nm}$. 

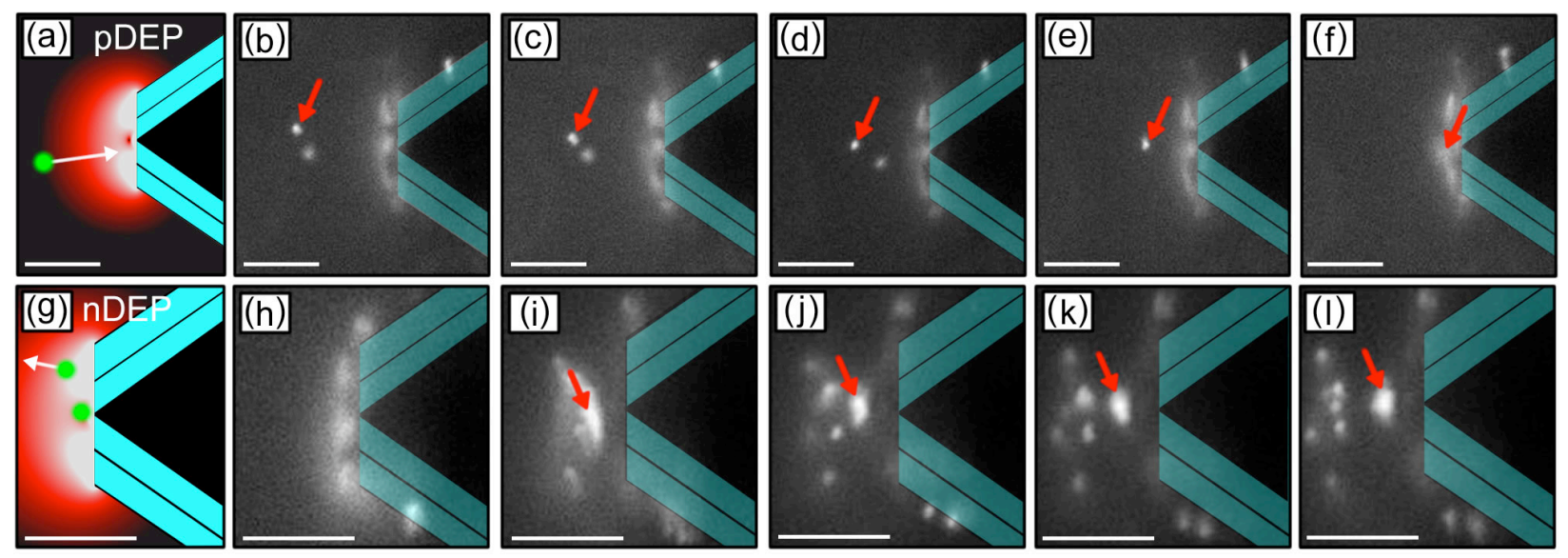

FIGURE 2. Attracting particles with pDEP and repelling and holding them with nDEP.

All scale bars $5 \mu \mathrm{m}$. (a) Schematic of a particle being drawn into the field maxima with pDEP. (b)-(f) A time sequence of fluorescence images depicting the bead marked with the red arrow being drawn into the tip with pDEP. A $10 \mathrm{~V}$ peak-to-peak voltage at $100 \mathrm{kHz}$ is applied to $V_{2}$ while $V_{l}=0$. Frames are separated by $200 \mathrm{~ms}$. The schematic cross section is overlayed in the approximate position of the triaxial probe as a guide to the eye. $(\mathrm{g})$ Schematic of one particle being repelled from the probe by nDEP and another being held in the nDEP trap. (h)-(l) A time sequence of fluorescence images depicting the triaxial probe repelling beads with nDEP. Just after the first frame, a $15 \mathrm{~V}$ peak-to-peak excitation is applied to $V_{2}$ at $5 \mathrm{MHz}$ while $V_{1}=0$. The majority of the beads are pushed back into suspension while the beads marked with the red arrow are held in the nDEP trap. Frames are separated by $200 \mathrm{~ms}$. 

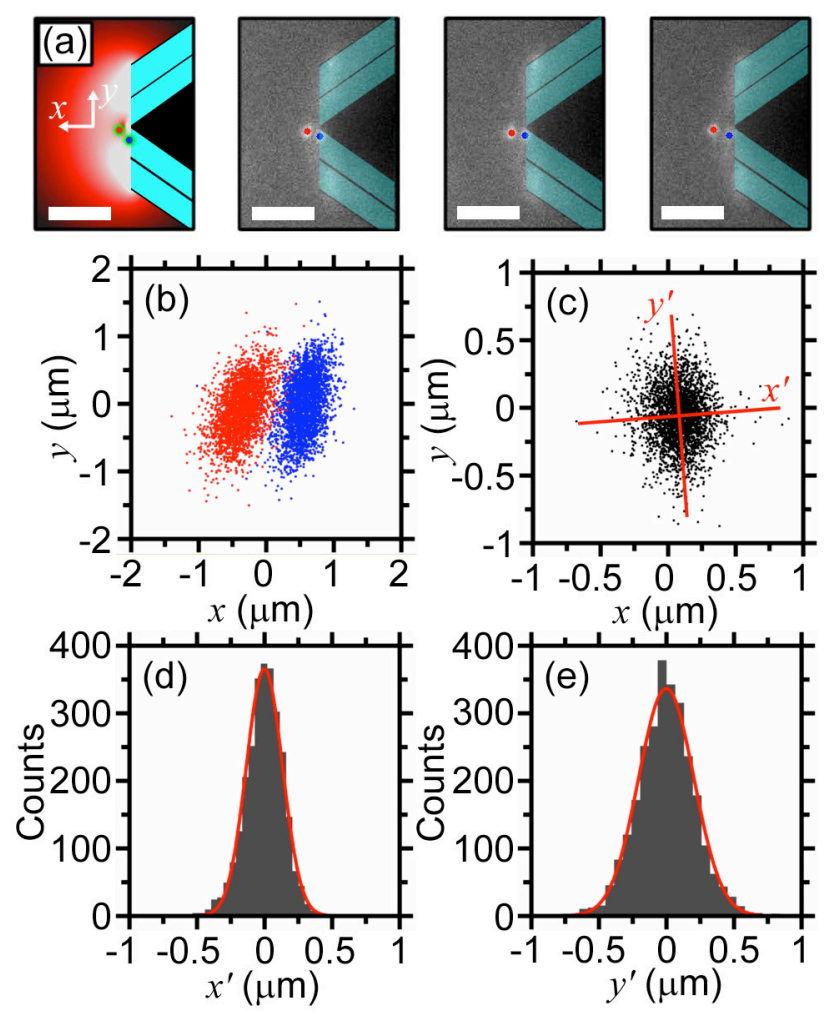

FIGURE 3. Determining the strength of the nDEP trap. (a) Schematic and three representative fluorescence images of a $100 \mathrm{~nm}$ radius bead trapped in the nDEP trap. The bead identified with the red dot is held in the nDEP trap while the bead identified with a blue dot is adhered to the tip. A schematic of the tip is superimposed in the approximate location of the tip as a guide to the eye. A $15 \mathrm{~V}$ peak-to-peak excitation at $1 \mathrm{MHz}$ is applied to $V_{2}$ while $V_{l}=0$. The scale bar $4 \mu \mathrm{m}$. These images are taken from a dataset of 5000 images used for the analysis. The full movie of this dataset is available as Video S1. (b) Scatter plots of the position of the bead in the trap (red dot) and the tip inferred from the position of an attached bead (blue dot). (c) Scatter plot of the separation between the trapped bead and the triaxial tip given by the separation of trapped bead/adhered bead pairs. The axes of tightest and loosest trapping are determined with principle component analysis and are shown on the plot as the $x^{\prime}$-axis and $y^{\prime}$-axis respectively. (d) Histogram of the spread of the trapped bead about the $x$ '-axis. A Gaussian fits the data very 
well with a standard deviation $\sigma_{x^{\prime}}=133 \mathrm{~nm}$. (e) Histogram of the spread of the trapped bead about the $y^{\prime}$-axis. A Gaussian fits the data very well with a standard deviation $\sigma_{y^{\prime}}=204 \mathrm{~nm}$. 

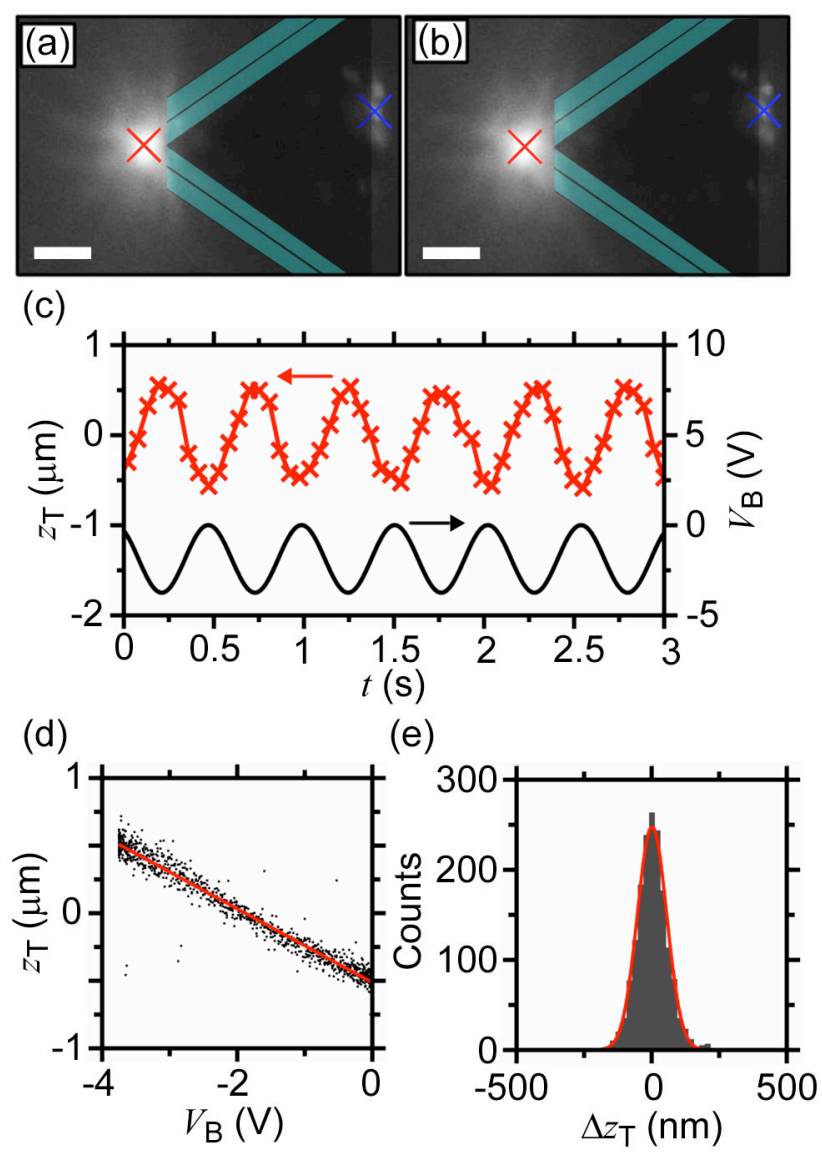

FIGURE 4. Controlling the position of a single trapped bead relative to the triaxial probe. (a) Fluorescence image of a $460 \mathrm{~nm}$ radius sphere held in the nDEP trap (red X). A bead adhered to the back of the probe (blue $\mathrm{X}$ ) is used as a reference to localize the tip. A $15 \mathrm{~V}$ peak-to-peak excitation at $5 \mathrm{MHz}$ is applied to $V_{2}$ while $V_{l}=0$. The scale bar is $4 \mu \mathrm{m}$. The full movie of this dataset is available as Video S2. (b) Now, the bead is held further from the tip as $7.5 \mathrm{~V}$ peak-topeak excitation at $5 \mathrm{MHz}$ is applied to $V_{2}$ while the same excitation is applied $180^{\circ}$ out of phase to $V_{l}$. (c) A control voltage $V_{B}$ is used to move the nDEP trap location smoothly (see Supporting Information). The position $z_{T}$ of the trap relative to the probe is determined by the distance between the trapped bead and the reference bead. (d) The position of the trap is a monotonic function of $V_{B}$ and we are able to move the bead $\sim 1 \mu \mathrm{m}$ relative to the probe. The data is well fit 
by a line, shown in red. (e) Histogram of the residual $z_{T}$ about the linear fit. A Gaussian fits the data very well and produces a standard deviation of $54 \mathrm{~nm}$. 